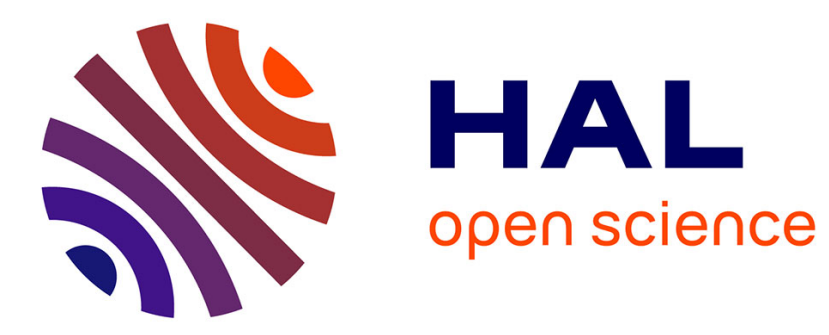

\title{
Key role of endothelium in the eNOS-dependent cardioprotection with exercise training
}

\author{
C. Farah, A. Nascimento, G. Bolea, G. Meyer, S. Gayrard, Alain Lacampagne, \\ Olivier Cazorla, C. Reboul
}

\section{- To cite this version:}

C. Farah, A. Nascimento, G. Bolea, G. Meyer, S. Gayrard, et al.. Key role of endothelium in the eNOSdependent cardioprotection with exercise training. Journal of Molecular and Cellular Cardiology, 2017, 102, pp.26 - 30. 10.1016/j.yjmcc.2016.11.008 . hal-01824256

\section{HAL Id: hal-01824256 \\ https://hal.umontpellier.fr/hal-01824256}

Submitted on 15 Dec 2019

HAL is a multi-disciplinary open access archive for the deposit and dissemination of scientific research documents, whether they are published or not. The documents may come from teaching and research institutions in France or abroad, or from public or private research centers.
L'archive ouverte pluridisciplinaire HAL, est destinée au dépôt et à la diffusion de documents scientifiques de niveau recherche, publiés ou non, émanant des établissements d'enseignement et de recherche français ou étrangers, des laboratoires publics ou privés. 


\title{
Key role of endothelium in the eNOS-dependent cardioprotection with exercise training
}

\author{
C. Farah ${ }^{\mathrm{a}, 1}$, A. Nascimento b,c,1, G. Bolea ${ }^{\text {b }}$, G. Meyer ${ }^{\mathrm{b}}$, S. Gayrard ${ }^{\mathrm{b}}$, A. Lacampagne ${ }^{\mathrm{a}}$, \\ O. Cazorla ${ }^{\mathrm{a}, *, 2}$, C. Reboul ${ }^{\mathrm{b}, * *, 2}$ \\ a CNRS UMR9214, INSERM U1046, Université de Montpellier, F-34295 Montpellier, France \\ b EA4278, LaPEC, Université d'Avignon, F-84000 Avignon, France \\ c CAPES Foundation, Ministry of Education of Brazil, Brasília/DF 70040-020, Brazil
}

\section{Keywords:}

Exercise

Cardioprotection

Ischemia-reperfusion

eNOS

Coronary artery

Myocytes

\begin{abstract}
A B S T R A C T
Modulation of endothelial nitric oxide synthase (eNOS) activation is recognized as a main trigger of the cardioprotective effects of exercise training on heart vulnerability to ischemia-reperfusion (IR). However, this enzyme is expressed both in coronary endothelial cells and cardiomyocytes and the contribution of each one to such cardioprotection has never been challenged. The aim of this study was to investigate the role of eNOS from the cardiomyocytes vs. the endothelium in the exercise cardioprotection. Male Wistar rats were assigned to a chronic aerobic training (Ex) (vs. sedentary group; Sed) and we investigated the role of eNOS in the effects of exercise on sensitivity to IR or anoxia-reoxygenation $(\mathrm{A} / \mathrm{R})$ at whole heart, isolated cardiomyocytes and left coronary artery (LCA) levels. We observed that exercise increased eNOS activation (Ser1177 phosphorylation) and protein Snitrosylation in whole heart but not at cardiomyocyte level, suggesting the specific target of endothelial cells by exercise. Consistently, in isolated cardiomyocytes submitted to the A/R procedure, exercise reduced cell death and improved cells contractility, but independently of the eNOS pathway. Next, to evaluate the contribution of endothelial cells in exercise cardioprotection, LCA were isolated before and after an IR procedure performed on Langendorff hearts. Exercise improved basal relaxation sensitivity to acetylcholine and markedly reduced the alteration of endothelium-dependent coronary relaxation induced by IR. Furthermore, inactivation of coronary endothelial cells activity just before IR, obtained with a bolus of Triton X-100, totally suppressed cardioprotective effects of exercise on both left ventricular functional recovery after IR and infarct size, whereas no effect of Triton X-100 was observed in Sed group. In conclusion, these results show that coronary endothelial cells rather than cardiomyocytes play a key role in the eNOS-dependent cardioprotection of exercise.
\end{abstract}

\section{Introduction}

Coronary artery diseases remain a leading cause of mortality in industrialized countries. Developing cardioprotective strategies to improve the management of myocardial ischemia-reperfusion (IR)

\footnotetext{
* Correspondence to: O. Cazorla, CNRS UMR-9214, Inserm U1046, Université de Montpellier "Physiologie et Médecine Expérimentale du Cœur et des Muscles PHYMEDEXP", CHRU Arnaud de Villeneuve, Bat. Castres Paulet, 371 avenue Gaston de Giraud, 34295 Montpellier Cedex 5, France.

** Correspondence to: C. Reboul, EA4278, LaPEC, Pôle sport et Recherche, Faculté des Sciences, technologies et Santé, Université d'Avignon, 74 rue Louis Pasteur, 84000 Avignon, France.

E-mail addresses: olivier.cazorla@inserm.fr (0. Cazorla), cyril.reboul@univ-avignon.fr (C. Reboul).

${ }^{1}$ Both authors contributed equally to this work.

2 Both senior co-authors.
}

injuries is a major challenge. Besides preventive strategies, exercise training is well recognized to reduce myocardial injuries following IR [1]. Possible explanations for exercise-induced cardioprotection were the increase of antioxidant buffering capacity [1,2] and preserved mitochondrial function [3]. More recently we [4] and others [5] have demonstrated the key role of the endothelial nitric oxide synthase (eNOS). eNOS catalyzes the synthesis of nitric oxide (NO) from L-arginine and oxygen, which triggers cardioprotection by the activation NO-cGMPPKG pathway, and/or by direct protein S-nitrosylation (SNO) [6]. Consistently, genetic deletion [5] or pharmacological inhibition [4] of eNOS abolished the exercise-induced cardioprotection. eNOS was first discovered and described in endothelial cells but is also expressed in cardiac myocytes [7]. In the heart of rodents, $80 \%$ of eNOS is localized in the coronary endothelium and participate in the relaxation of vascular smooth muscle cells. The remaining $20 \%$ of eNOS are located in the cardiomyocytes [8]. Whether exercise activates eNOS both in 
endothelial cells and in cardiomyocytes to protect the heart during IR has never been investigated.

In this work, we aimed to determine the relative contribution of eNOS expressed in the cardiomyocytes vs. in the endothelial cells in cardioprotection afforded by exercise training during IR.

\section{Materials and methods}

All investigations conformed to the European Parliament Directive 2010/63/EU and approved by the research ethics committee of Marseille (n. 00,322.03).

\subsection{Animals and experimental groups}

Male Wistar rats (Charles River Laboratories, France) (12 weeks old, $n=90$ ) were randomly assigned to sedentary (Sed) or exercised (Ex) group. Rats were exercised for 5 weeks on a motor treadmill according to a training protocol previously reported to reduce heart vulnerability to IR ( $25 \mathrm{~m} / \mathrm{min} ; 45 \mathrm{~min} /$ day; 5 days/week) [4].

\subsection{Ischemia-reperfusion procedure on isolated perfused heart}

Ischemia-reperfusion procedure was performed as previously described on a Langendorff apparatus [4]. Briefly, after stabilization a global ischemia was performed for $30 \mathrm{~min}$, followed by reperfusion (10 or $120 \mathrm{~min}$ ). The left anterior coronary artery was dissected at the end of stabilization or after $10 \mathrm{~min}$ of post-ischemic reperfusion to assess coronary endothelium-dependent relaxation in isolated rings ( $n=10$ / group) (see below). The left ventricular developed pressure (LVDP; MP35, BioPac System Inc) was measured after 10 min of reperfusion to match with the coronary artery function evaluation. Infarct size was assessed after $120 \mathrm{~min}$ of reperfusion with tryphenyltetrazolium-chloride (TTC), as previously described [4]. In some hearts, the endothelium was inactivated by perfusing a Triton X-100 bolus solution $(0.25 \%)$ at the end of the stabilization period, as previously described [9]. This procedure has been shown to abolish endothelium-dependent signaling with minimal effects on myocardial function [9].

\subsection{Anoxia-reoxygenation procedure and isolated cardiomyocytes contractility}

Single cardiomyocytes were isolated from the left ventricle by enzymatic digestion as previously described [10]. Myocytes were placed in a Petri dish and placed in an anoxic chamber $\left(\mathrm{O}_{2}\right.$ level $\left.\sim 2 \%\right)$ for $60 \mathrm{~min}$, followed by a 60 min reoxygenation in ambient air $\left(\mathrm{O}_{2} \sim 19.4 \%\right)$. This anoxia-reoxygenation $(A / R)$ experiment was carried out in the presence or absence of eNOS inhibitor L-NAME ( $100 \mu \mathrm{M}$; incubation during A/ $\mathrm{R})$. Cell survival was evaluated with Malassez hemocytometer counting before and after $A / R$ procedure, and cell shortening was measured by recording sarcomere length (SL) variations (Ionoptix system; Hilton) at $1 \mathrm{~Hz}$ electrical field stimulation.

\subsection{Isolated left anterior coronary artery reactivity}

Left coronary arteries (LCA) were isolated and $2 \mathrm{~mm}$ in length segments were mounted in a small vessel dual chamber wire-myograph for measurement of isometric tension (wire myograph system Myo2, EMKA). After a 30 min equilibration period in oxygenated KrebsHenseleit solution (KHS, in mM: $115 \mathrm{NaCl}, 25 \mathrm{NaHCO}_{3}, 4.7 \mathrm{KCl}, 1.2$ $\mathrm{MgSO}_{4}, 7 \mathrm{H}_{2} \mathrm{O}, 2.5 \mathrm{CaCl}_{2}, 1.2 \mathrm{KH}_{2} \mathrm{PO}_{4}, 11.1$ glucose and $\left.0.01 \mathrm{Na}_{2} \mathrm{EDTA}\right)$ at $37^{\circ} \mathrm{C}$ and $\mathrm{pH}$ 7.4, segments were stretched to their optimal lumen diameter for active tension development. LCA were exposed to a high $\mathrm{K}^{+}$ solution ( $\mathrm{K}^{+}-\mathrm{KHS}, 120 \mathrm{mM}$ ) to check functional integrity. Then endothelium integrity was assessed by measuring the endothelium-dependent vasorelaxation to $\mathrm{ACh}\left(10^{-6} \mathrm{M}\right)$ in pre-contracted coronary artery rings (U46619, $1 \mu \mathrm{M}$ ), and a concentration-response curve to ACh $\left(10^{-9}\right.$ to $\left.10^{-4} \mathrm{M}\right)$ was performed.

\subsection{Western blot analysis}

Proteins from LV or isolated cardiomyocytes pellet were separated by SDS-polyacrylamide gel electrophoresis (SDS-PAGE) and transferred onto PVDF membranes. Membranes were incubated with primary antibodies: anti-mouse eNOS (BD Transduction Laboratory), anti-mouse eNOS-PSer1177 (BD Transduction Laboratory), anti-rabbit GAPDH (Santa Cruz). Immunodetection was carried out using ECL Plus system (Luminata ${ }^{\mathrm{TM}}$ Forte Western HRP substrate, Millipore Corporation) and membranes were then exposed to X-ray films for revelation. Protein content was expressed relative to the GAPDH content.

\subsection{Measurement of protein S-nitrosylation}

S-nitrosylation was determined using the modified S-nitrosylation switch assay that uses a non-biological iodo TMT Reagent for labeling (Pierce S-nitrosylation Western blot kit, Thermo Fischer Scientific). Snitrosylated proteins in LV homogenates or isolated cardiomyocytes were detected by Western blot using an anti-TMT antibody (Thermo Fischer Scientific).

\subsection{Measurement of nitrite}

Quantification of nitrite in LV or cardiomyocyte lysates was carried out using the Measure-iT ${ }^{\mathrm{TM}}$ High-Sensitivity Nitrite Assay Kit (Invitrogen) according to the manufacturer's instructions.

\subsection{Statistical analysis}

Data were expressed as the mean \pm S.E.M. One way or multiple analysis of variance (ANOVA) with or without repeated measures were used to compare multiple experimental conditions, followed by the post-hoc Bonferroni adjusted $t$-test. Binomially distributed variables such as cell death were analyzed using non-parametric Yates' chi square test. A value of $p<0.05$ was considered statistically significant.

\section{Results and discussion}

The key role of eNOS in exercise-induced cardioprotection has been recently reported $[4,5]$. However, in those studies inactivation of eNOS was performed on the whole heart, making impossible to discriminate the specific involvement of cardiomyocytes and/or endothelial cells in the cardioprotection. Here, to investigate the relative contribution of the one or the other we compared the effects of exercise on whole heart and isolated cardiomyocytes levels. In basal condition, exercise increased in the whole heart the amount of eNOS phosphorylation on its activation site (eNOS-P ${ }^{\text {Ser1177}}$ ) without affecting eNOS expression (Fig. 1A), which is consistent with previous report [4]. However, on cardiomyocytes, exercise training had no effect on eNOS-P ${ }^{\text {Ser1177 }}$ and even reduced eNOS expression (Fig. 1B). Cardioprotection by exercise training mainly depends on eNOS-dependent increase of NO metabolites, nitrite and S-nitrosothiols storage [5]. Indeed, since eNOS is not active during ischemia [4] the NO metabolites storage constitutes a pool of NO available during IR to active protective pathways. In line with our observations on eNOS status, we observed that NO metabolites, protein S-nitrosylation (Fig. 1C) and nitrites (Fig. 1E), were increased with exercise in the whole heart but not in the cardiomyocytes level (Fig. 1D-E). Considering that in the heart eNOS is mainly expressed in endothelial cells [8], our results strongly suggest that such differences observed between whole hearts and cardiomyocytes reflect the modulation of eNOS on endothelial cells. Therefore, it seems that exercise training enhances cardiac eNOS-NO pathway mostly by targeting eNOS from the vascular endothelium layer. 

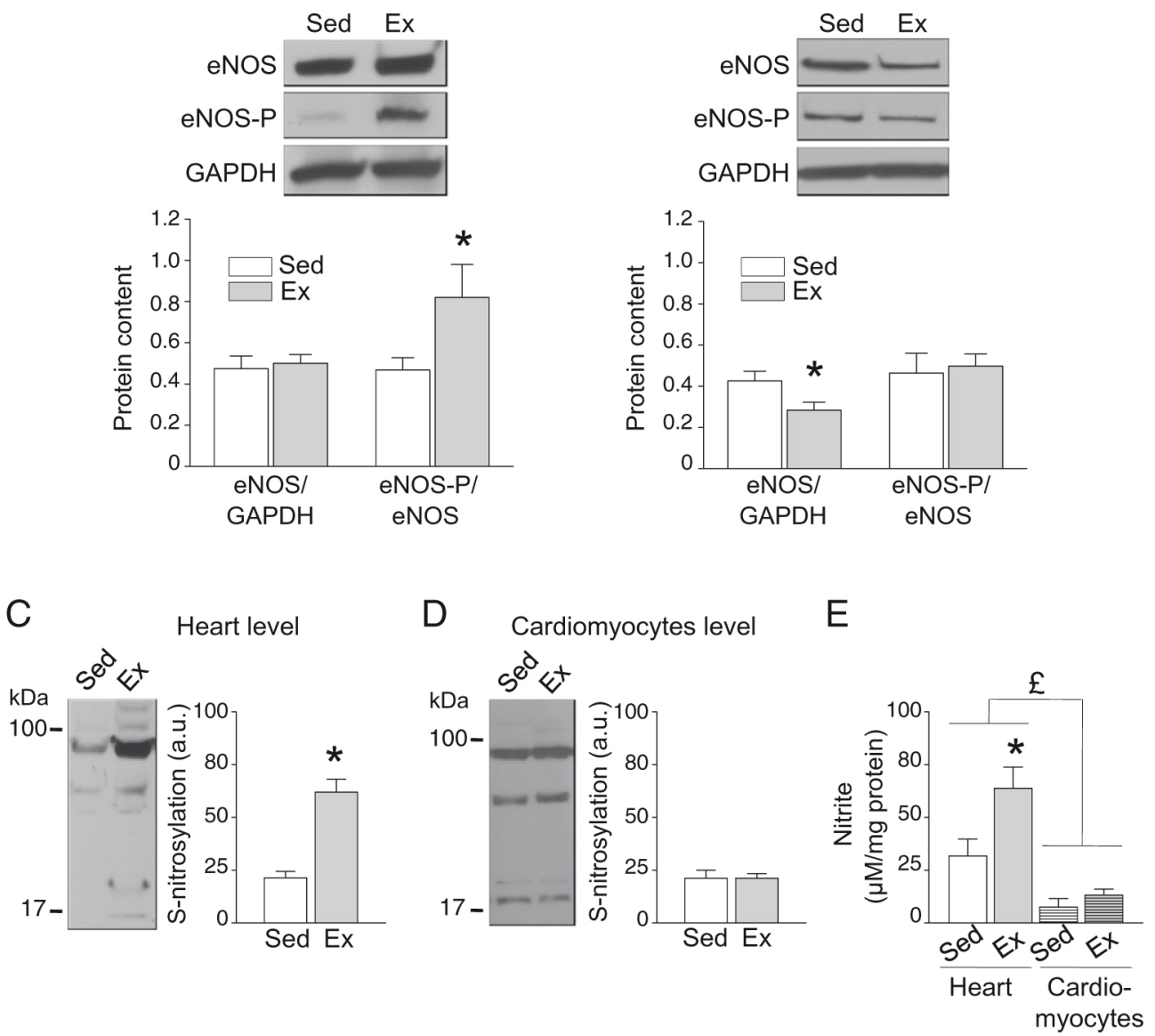

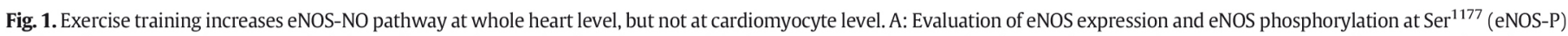

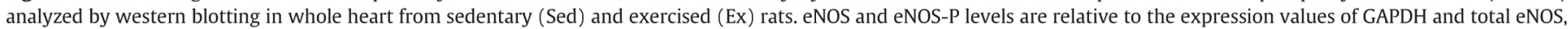

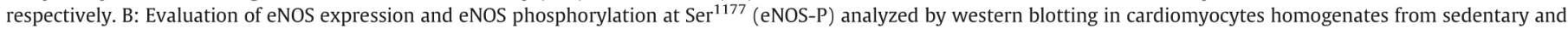

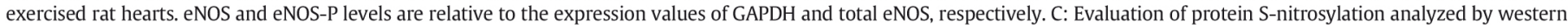

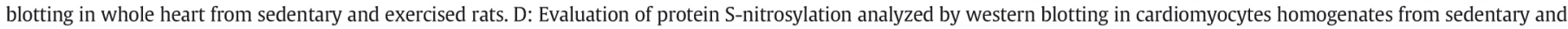

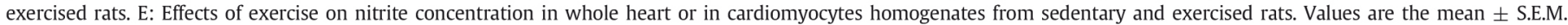
$* p<0.05$ sedentary group vs. exercised group, $£ p<0.05$ whole heart vs. cardiomyocytes. $N=5$ rats per group.

Next, we challenged the efficacy of exercise to protect by an eNOSdependent way the cardiomyocytes or the coronary arteries against ischemia-reperfusion events. Cardiomyocytes isolated from sedentary and exercised hearts were exposed to A/R stress. Then cell contractility and cell death were evaluated. In basal condition, our model of exercise training had no effect on cardiomyocyte sarcomere length shortening (Fig. 2A-B). However, following A/R, the cell shortening amplitude of the surviving sedentary cardiomyocytes was decreased (Fig. 2A-B), whereas this hypo-contractility following $\mathrm{A} / \mathrm{R}$ was not observed in exercised cardiomyocytes. Moreover about $53 \%$ of the cardiomyocytes isolated from sedentary hearts died after $\mathrm{A} / \mathrm{R}$ stress for only $\approx 30 \%$ of exercised myocytes (Fig. 2C). Thus, in line with one previous study [11], we reported that exercise reduces myocyte sensitivity to A/R. The involvement of eNOS in this cardioprotection was tested by the presence of L-NAME before and during A/R. While we have previously reported that L-NAME blunted the beneficial effects of exercise training on heart sensitivity to IR [4], in cardiomyocytes L-NAME had no effect on cell death and contractility whatever the experimental group (Fig. $2 \mathrm{~A}-\mathrm{C}$ ). Therefore, we show here that exercise training protects the cardiomyocytes against A/R stress independently of the eNOS pathway. Others signaling pathways should certainly be involved in the beneficial effects of exercise, such as enhancement of calcium homeostasis [10] and increased antioxidant capacity [1,2]. To note, even if our results strongly suggest that exercise-induced cardioprotection at the level of cardiomyocytes does not involve eNOS-NO pathway, we cannot exclude that $\mathrm{A} / \mathrm{R}$ on isolated of cardiomyocytes could impact this pathway differently than IR performed on whole heart. However, the hypoxia-reoxygenation method of cardiomyocytes had been previously validated as a relevant approach to investigate cardiac myocyte molecular and cellular mechanisms involved in the heart IR injury [11]. Indeed, in line with our results, Portal et al. [11] observed that exercise is able to protect the heart during IR and also the isolated cardiomyocytes during hypoxia-reoxygenation (with a $\mathrm{O}_{2} \%$ similar to our $\mathrm{A} / \mathrm{R}$ method). The authors conclude that $\mathrm{A} / \mathrm{R}$ on isolated cardiomyocytes shows several features of IR on whole heart.

Considering the potential key role of the endothelial layer in exercise-induced cardioprotection, we next explored the impact of exercise on the coronary artery endothelium function during IR. The left coronary arteries (LCA) were isolated before and after cardiac IR and the eNOS-dependent response of the endothelium to ACh was assessed in pre-contracted LCA. In line with previous work showing that exercise improves coronary endothelial function in an eNOS-dependent manner [12], we observed in basal condition, i.e. before IR, a leftward shift of the relaxation-[ACh] curve in LCA of exercised hearts compared to the sedentary ones (Fig. 2D), indicating that exercise increases the sensitivity of LCA to ACh. After IR, the LCA response to ACh was reduced in both sedentary and exercised hearts (Fig. 2D). However, a major result was that vasorelaxation in response to ACh was obviously more preserved in exercised LCA compared to sedentary. Indeed, the maximal response to $10^{-4} \mathrm{M}$ of ACh was two times higher after IR in exercised LCA than 
A

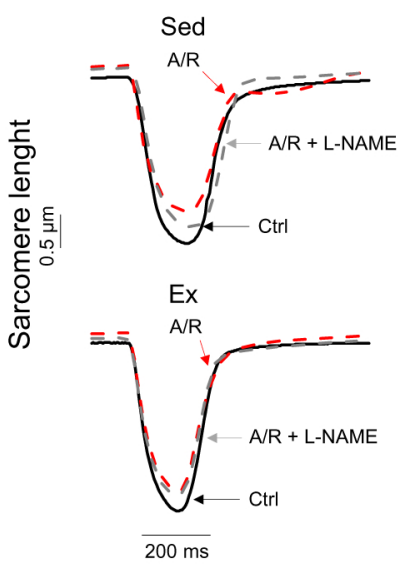

C

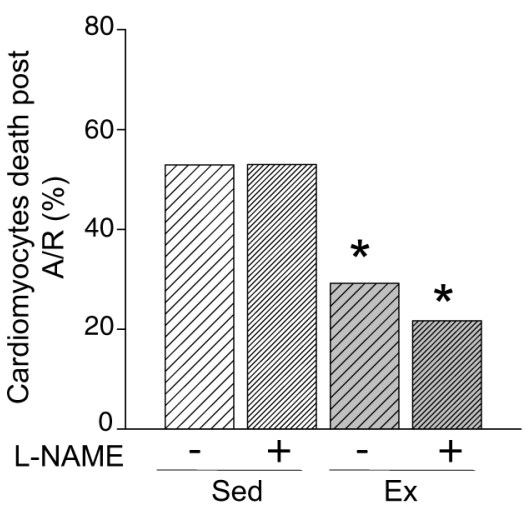

$\mathrm{E}$

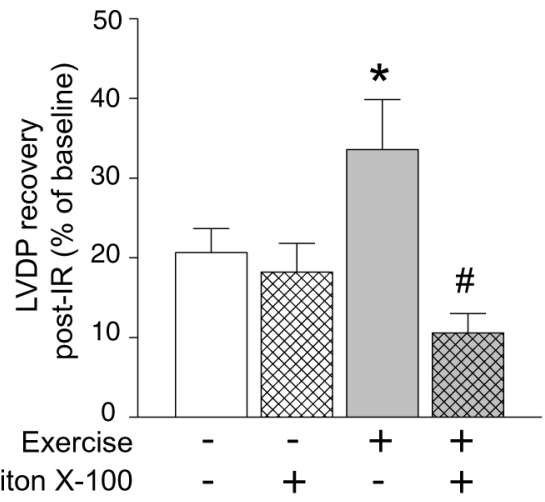

B
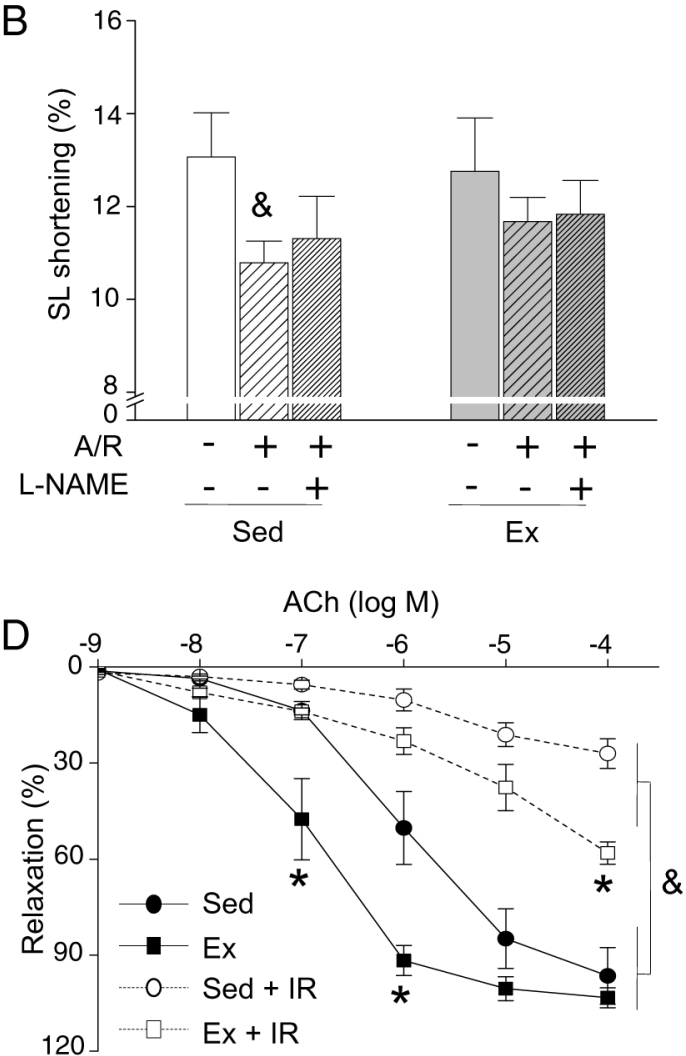

\&

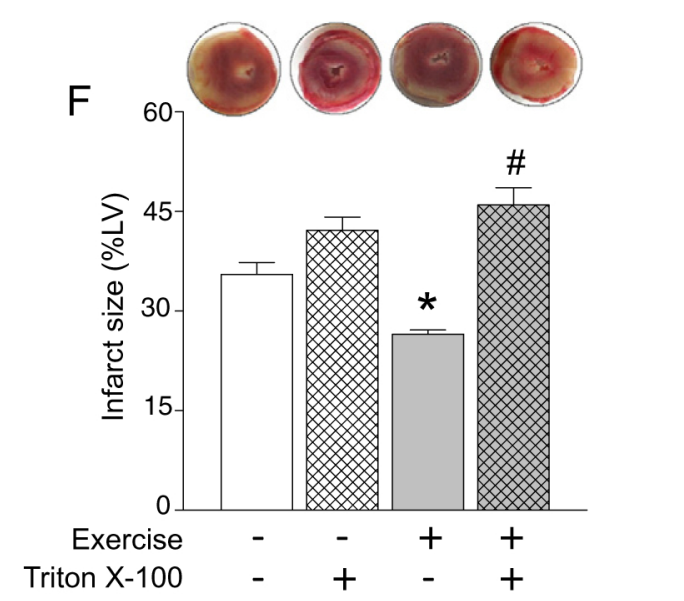

Fig. 2. Coronary endothelium plays a key role in exercise-induced cardioprotection. A: Representative recordings of cell shortening at $1 \mathrm{~Hz}$ in sedentary (Sed; top panel) and exercised (Ex; bottom panel) isolated cardiomyocytes in basal condition (black full line), or submitted to anoxia-reoxygenation (A/R) procedure ( $1 \mathrm{~h}$ anoxia/1 h reoxygenation) in presence (grey dashed line) or not of L-NAME (N-nitro-L-arginine methyl ester; a NOS inhibitor) (red dashed line). B: Effect of exercise on cardiomyocytes contractility measured by the amplitude of sarcomere length (SL) shortening, in basal condition or after the A/R procedure in presence or not of L-NAME. C. Effects of exercise on cardiomyocytes death after A/R procedure, in presence or not of L-NAME. Results are expressed in percentage of dead cells relative to the total number of cells. D: Evaluation of endothelium-dependent relaxation to cumulative concentrations of acetylcholine (ACh) $\left(10^{-9}\right.$ to $\left.10^{-4} \mathrm{M}\right)$ of isolated left coronary artery rings from sedentary and exercised rat hearts in basal condition (Sed and Ex) or after 30 min of total global ischemia and 10 min of reperfusion (Sed + IR; Ex + IR). Data are expressed in percentage of pre-contraction with U46619. E: Assessment of left ventricular developed pressure (LVDP) measured after $30 \mathrm{~min}$ of global ischemia and $10 \mathrm{~min}$ of reperfusion in hearts from sedentary and exercised rats, perfused or not with a bolus of Triton X-100 (administered at the stabilization period; $0.25 \%$ ). LVDP is expressed as percentage of the baseline value. F: Evaluation of infarct size in hearts from exercised and sedentary rats, treated or not with Triton X-100, following $30 \mathrm{~min}$ of ischemia and $120 \mathrm{~min}$ of reperfusion. Top panel: Heart sections stained with triphenyltetrazolium chloride to show the infarct size. Infarct size is expressed as percentage of the left ventricular section area. Values are the mean \pm S.E.M. ${ }^{*} p<0.05$ sedentary group vs. exercised group, \& $p<0.05$ vs. baseline condition, \# $p<0.05$ Triton X-100 vs. homolog group. $N=5$ rats per group for isolated cardiomyocyte (10 cells/rat) and isolated left coronary artery; $N=10$ rats per group for isolated heart experiments.

in sedentary ones (Fig. 2D). To the best of our knowledge, this constitutes the first experiments showing that exercise training is also able to protect the endothelial eNOS-dependent vasorelaxation of coronary arteries during IR. To date, the beneficial effects of exercise on the function of coronary arteries have been only investigated in models of chronic coronary occlusion, i.e. in vessels which were not submitted to
IR $[13,14]$. Then, to further explore the contribution of the endothelial cells in the protective effects of exercise, we chemically destroyed them with a bolus of Triton X-100 in isolated-perfused hearts. A key result of our work is that inhibition of the endothelium activity and thus of the endothelial eNOS/NO pathway signaling during IR totally abolished the cardioprotective effects of exercise training assessed on the LV 
functional recovery (Fig. 2E) and the infarct size (Fig. 2F). Meantime Triton X-100 had no significant effect in sedentary hearts functional recovery (Fig. $2 \mathrm{E}$ ) and only tended to increase infarct size without reaching significance (Fig. 2F). Altogether, our results are the first to demonstrate that the key role of eNOS signaling in exercise-induced cardioprotection during IR involves the endothelial coronary layer. Even so, we reported any effect of exercise on coronary perfusion during IR (coronary output after $10 \mathrm{~min}$ of reperfusion: Sed: $5.2 \pm 0.3 \mathrm{ml} / \mathrm{min}$; Ex: $5.2 \pm$ $0.6 \mathrm{ml} / \mathrm{min}$; NS), which suggests that the beneficial effects of exercise on eNOS-dependent pathway in endothelial cells may protect through a paracrine effect of NO on cardiomyocytes. Such cross-talk between endothelial cells and cardiomyocytes has already been reported in the regulation of cardiomyocyte function. Especially, Leucker et al. [15] showed that cardiomyocytes were less sensitive to $\mathrm{A} / \mathrm{R}$ when co-cultured with endothelial cells pre-conditioned to increase NO availability. Moreover, increased NO pathway in cardiomyocyte is well recognized to protect against IR injuries by improving excitation-contraction coupling with protective mechanisms on sarcomeric and calcium handling proteins [7, for review]. Thus in our model, we could propose that increased NO metabolites storage and signaling in endothelial cells with exercise should promote a paracrine liberation of NO, which then contributes to protect the heart against IR. Further studies will be needed to investigate how this cross-talk contribute to the central role of eNOS in exercise-induced cardioprotection.

In conclusion, we show that the endothelium is a key trigger of the eNOS/NO-dependent cardioprotection afforded by exercise training. Such results enable a new understanding of a key cardioprotective strategy and improve our knowledge of eNOS cardioprotective signaling pathway against ischemia-reperfusion injuries, showing the potential role of coronary endothelium as a paracrine organ to protect the heart during ischemic stress.

\section{Disclosures}

None.

\section{References}

[1] S.K. Powers, J.C. Quindry, A.N. Kavazis, Exercise-induced cardioprotection against myocardial ischemia-reperfusion injury, Free Radic. Biol. Med. 44 (2008) 193-201
[2] J.P. French, K.L. Hamilton, J.C. Quindry, Y. Lee, P.A. Upchurch, S.K. Powers, Exerciseinduced protection against myocardial apoptosis and necrosis: MnSOD, calciumhandling proteins, and calpain, FASEB J. 22 (2008) 2862-2871.

[3] R.J. Alleman, A.M. Tsang, T.E. Ryan, D.J. Patteson, J.M. McClung, E.E. Spangenburg, et al., Exercise-induced protection against reperfusion arrhythmia involves stabilization of mitochondrial energetics, Am. J. Physiol. Heart Circ. Physiol. 310 (10) (2016) H1360-H1370.

[4] C. Farah, A. Kleindienst, G. Bolea, G. Meyer, S. Gayrard, B. Geny, et al., Exercise-induced cardioprotection: a role for eNOS uncoupling and NO metabolites, Basic Res. Cardiol. 108 (2013) 389.

[5] J.W. Calvert, M.E. Condit, J.P. Aragón, C.K. Nicholson, B.F. Moody, R.L. Hood, et al., Exercise protects against myocardial ischemia-reperfusion injury via stimulation of $\beta(3)$-adrenergic receptors and increased nitric oxide signaling: role of nitrite and nitrosothiols, Circ. Res. 108 (2011) 1448-1458.

[6] J.N. Simon, D. Duglan, B. Casadei, R. Carnicer, Nitric oxide synthase regulation of cardiac excitation-contraction coupling in health and disease, J. Mol. Cell. Cardiol. 73 (2014) 80-91.

[7] J.L. Balligand, L. Kobzik, X. Han, D.M. Kaye, L. Belhassen, D.S. O'Hara, et al., Nitric oxide-dependent parasympathetic signaling is due to activation of constitutive endothelial (type III) nitric oxide synthase in cardiac myocytes, J. Biol. Chem. 270 (1995) 14582-14586.

[8] A. Gödecke, T. Heinicke, A. Kamkin, I. Kiseleva, R.H. Strasser, U.K. Decking, et al., Inotropic response to beta-adrenergic receptor stimulation and anti-adrenergic effect of ACh in endothelial NO synthase-deficient mouse hearts, J. Physiol. 532 (2001) 195-204.

[9] C. Yang, M.A.H. Talukder, S. Varadharaj, M. Velayutham, J.L. Zweier, Early ischaemic preconditioning requires Akt- and PKA-mediated activation of eNOS via serine1176 phosphorylation, Cardiovasc. Res. 97 (2013) 33-43.

[10] Y. Aït Mou, C. Reboul, L. Andre, A. Lacampagne, O. Cazorla, Late exercise training improves non-uniformity of transmural myocardial function in rats with ischaemic heart failure, Cardiovasc. Res. 81 (2009) 555-564.

[11] L. Portal, V. Martin, R. Assaly, A. d'Anglemont de Tassigny, S. Michineau, et al., A model of hypoxia-reoxygenation on isolated adult mouse cardiomyocytes: characterization, comparison with ischemia-reperfusion, and application to the cardioprotective effect of regular treadmill exercise, J. Cardiovasc. Pharmacol. Ther. 18 (2013) 367-375.

[12] M.H. Laughlin, D.K. Bowles, D.J. Duncker, The coronary circulation in exercise training, AJP Heart Circ. Physiol. 302 (2012) H10-H23.

[13] K.L. Griffin, C.R. Woodman, E.M. Price, M.H. Laughlin, J.L. Parker, Endothelium-mediated relaxation of porcine collateral-dependent arterioles is improved by exercise training, Circulation 104 (2001) 1393-1398.

[14] M. Zhou, R.J. Widmer, W. Xie, A. Jimmy Widmer, M.W. Miller, F. Schroeder, et al., Effects of exercise training on cellular mechanisms of endothelial nitric oxide synthase regulation in coronary arteries after chronic occlusion, Am. J. Physiol. Heart Circ. Physiol. 298 (2010) H1857-H1869.

[15] T.M. Leucker, Z.-D. Ge, J. Procknow, Y. Liu, Y. Shi, M. Bienengraeber, D.C. Warltier, et al., Impairment of endothelial-myocardial interaction increases the susceptibility of cardiomyocytes to ischemia/reperfusion injury, PLoS One 8 (2013), e70088. 